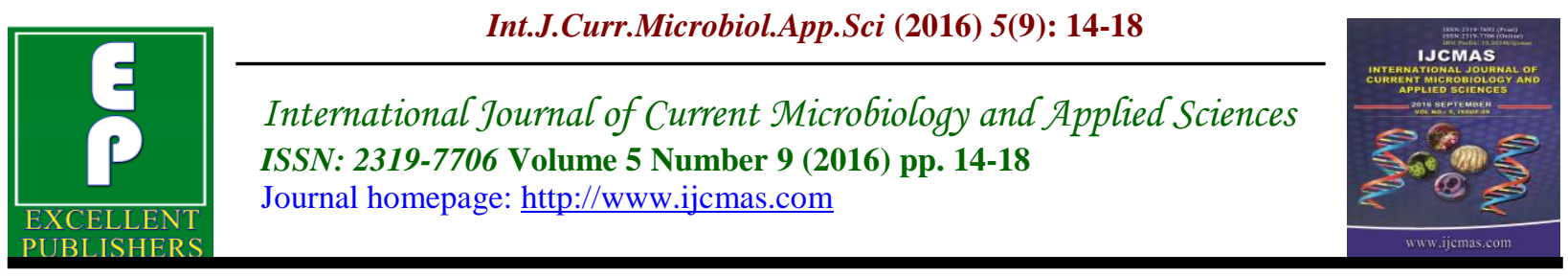

Original Research Article

http://dx.doi.org/10.20546/ijcmas.2016.509.002

\title{
Seroprevalence of Rickettsial Diseases in a Tertiary Care Hospital
}

\author{
Mita D. Wadekar ${ }^{1 *}$ and N.B. Swaroop Rani \\ ${ }^{1}$ Assistant Professor, ${ }^{2}$ Associate Professor, Department of Microbiology, \\ Subbaiah Institute of Medical Sciences, Shimoga, Karnataka, India \\ *Corresponding author
}

\begin{tabular}{|c|c|}
\hline & A B S T R A C T \\
\hline & \multirow{6}{*}{$\begin{array}{l}\text { Rickettsial diseases are still prevalent in most of the countries including India and } \\
\text { are also classified under the causes of pyrexia of unknown origin (PUO). Early } \\
\text { signs and symptoms of these infections are nonspecific making diagnosis more } \\
\text { difficult. If untreated mortality may be as high as } 30-35 \% \text {. Though Weil Felix test } \\
\text { is less sensitive but still it serves as a useful and cheapest available screening tool } \\
\text { for the laboratory diagnosis of rickettsial diseases. A prospective study was carried } \\
\text { out for a period of } 6 \text { months from November } 2015 \text { to April } 2016 \text { in a tertiary-care } \\
\text { hospital, Shimoga, Karnataka, India. The serum samples from } 277 \text { PUO cases } \\
\text { which included patients of all age group and from both Out Patient Department } \\
\text { (OPD) and In Patient Department (IPD), were subjected to Weil-Felix test } \\
\text { (PROGEN, Tulip Diagnostics (P) Ltd., Verna, Goa, India). The test was performed } \\
\text { according to the manufacturer's instructions. Serum samples positive in the slide } \\
\text { test were confirmed with the tube test. Titers of more than } 1: 160 \text { for OX-K and } \\
\text { more than 1:80 for OX-2 and OX-19 were considered significant. Of } 277 \text { samples, } \\
\text { rickettsial diseases were detected in } 73(26.35 \%) \text { samples. Seropositivity was higher } \\
\text { among male subjects 52(71.23\%) when compared with female subjects } \\
21(28.77 \%) \text {. All positive cases were in } 1-18 \text { age group. Prevalence of rickettsial } \\
\text { diseases } 73(26.35 \%) \text { is significantly high, especially in children's and hence should } \\
\text { be included in the differential diagnosis of PUO. }\end{array}$} \\
\hline Keywords & \\
\hline $\begin{array}{l}\text { Rickettsial } \\
\text { diseases, } \\
\text { PUO, } \\
\text { Weil-Felix } \\
\text { test. }\end{array}$ & \\
\hline Article Info & \\
\hline & \\
\hline & \\
\hline
\end{tabular}

\section{Introduction}

Rickettsiae comprise of group of small nonmotile gram negative coccobacilli and are obligate intracellular, transmitted by arthropod vector like lice, fleas, ticks (Raghu Kumar et al., 2015). Species of Rickettsia can be categorized into spotted fever, typhus, and scrub typhus groups based on clinical manifestations (Tay et al., 2003). Rickettsial infections are one of the important causes of pyrexia of unknown origin (PUO) and this needs to be differentiated from other common febrile illnesses like enteric fever, malaria, dengue etc (Veena Mittal et al., 2012). They have a global distribution and are reported from almost all parts of India like Jammu and Kashmir, Himachal Pradesh, Uttaranchal, Rajasthan, Assam, West Bengal, Maharashtra, Kerala and Tamil Nadu (Anuradha Sood et al., 2013). 
Rickettsial diseases widely vary in severity from self limited mild illnesses to fulminating life threatening infections. These diseases usually manifest as fever, rashes and vasculitis (Suchitra Shenoy et al., 2015). Although clinical manifestations of rickettsial infections are well documented, recent studies in Asian countries have reported complications such as gastrointestinal manifestations, tinnitus, and hepatitis syndromes (Tanveer Nawab et al., 2015). Mortality due to these infections is reported to occur in $1 \%$ to $30 \%$ of untreated cases (Narendra rathi et al., 2010; Prabhakaran et al., 2010).

As no single laboratory finding is specific for early diagnosis, treatment needs to be started empirically on clinical and epidemiological suspicion. The greatest challenge to the clinician is the diagnostic dilemma posed by these infections early in their clinical course when antibiotic therapy is most effective. Microimmunofluorescence, latex agglutination, indirect hemagglutination, immunoperoxidase assay, and enzyme-linked immunosorbent assay are various serological tests available. Immunofluorescence assay (IFA) is the "gold standard" technique (Mahajan et al., 2006). Even though the sensitivity and specificity of Weil Felix test is low, in most of small laboratories, this method is the only one available. This study was conducted to know the prevalence of rickettsial diseases.

\section{Materials and Methods}

Prospective study of 6 months from November 2015 to April 2016 was done in a tertiary-care hospital, Shimoga, Karnataka, India. Rickettsial diseases were diagnosed by Weil- Felix test (PROGEN, Tulip Diagnostics (P) Ltd., Verna, Goa, India). Total of 277 serum samples from PUO cases were subjected to Weil-Felix test. Test is based on the principle that some strains of Proteus share common somatic constituents with certain species of Rickettsia. Sera from patients infected with Rickettsia will, therefore, produce agglutination with Proteus antigen suspensions. Antigen suspension of Proteus OX19 antigen reacts strongly with the sera of patients with typhus group rickettsiae and rocky mountain spotted fever, Proteus OX2 with the sera of patients with spotted fever infections, while the Proteus OXK with the sera of patients infected with scrub typhus. Serum samples positive in the slide test were confirmed with the tube test. Titers of more than 1:80 for OX2 and OX19 and more than 1:160 for OXK were considered diagnostically significant.

\section{Result and Discussion}

Of 277 samples, rickettsial diseases were detected in $73(26.35 \%)$ samples. Seropositivity was higher among male subjects 52(71.23\%) when compared with female subjects 21(28.77\%). All positive cases were in 1-18 age group. 13 samples were reactive to OX 2 only, 5 to OX K only but none to OX 19 individually. When reactivity was seen towards two or more antigens in the same serum sample, combination of antigens demonstrated it are OX $19+$ OX 2, OX $19+$ OX K, OX $2+$ OX $\mathrm{K}$ and OX $19+$ OX $2+$ OX K. Maximum (27) reactivity was in combination OX $19+$ OX 2 + OX K.

Rickettsial disease is an acute infectious disease transmitted to humans by ticks, mites or lice. They are underdiagnosed because of their varied presentation and lack of availability of reliable, specific laboratory tests. Rickettsial fever can be present with conjunctivitis, generalized oedema, meningoencephalitis and pupura fulminance. 
In the present study, rickettsial diseases were detected in $73(26.35 \%)$ samples with male predominance $52(71.23 \%)$ which is similar to studies done by Raghu Kumar KG et al., (2015) and Anuradha Sood et al., (2013). Maximum (27) reactivity was in combination OX $19+$ OX 2 + OX K which is consistent with study done by Ganavalli Subramanya Ajantha et al., (2013). Rickettsial disease is a very less known cause of fever of unknown origin. They are re-emerging with increased reports from different parts of the world (Ponugoti et al., 2015). The Rickettsial diseases are endemic in Southeast Asia, Northern Australia and pacific islands. Amongst Rickettisiosis scrub typhus is most common and is endemic in the tropical and subtropical regions of the Asian continent. The presence of rickettsial diseases in India have been documented in subhimalayan region of the country (Anuradha et al., 2005).

Table.1 Total PUO cases with their reactivity

\begin{tabular}{|c|c|c|}
\hline $\begin{array}{c}\text { Positive samples } \\
\text { No. (\%) }\end{array}$ & $\begin{array}{c}\text { Negative samples } \\
\text { No. (\%) }\end{array}$ & $\begin{array}{c}\text { Total } \\
\text { No. (\%) }\end{array}$ \\
\hline $73(26.35)$ & $204(73.65)$ & 277 \\
\hline
\end{tabular}

Table.2 Sex -wise distribution of total cases

\begin{tabular}{|c|c|c|c|}
\hline Sex & $\begin{array}{c}\text { Positive samples } \\
\text { No. (\%) }\end{array}$ & $\begin{array}{c}\text { Negative samples } \\
\text { No. (\%) }\end{array}$ & $\begin{array}{c}\text { Total } \\
\text { No. (\%) }\end{array}$ \\
\hline Male & $52(71.23)$ & $116(56.87)$ & $168(60.65)$ \\
\hline Female & $21(28.77)$ & $088(43.13)$ & $109(39.35)$ \\
\hline Total & $73(100)$ & $204(100)$ & $277(100)$ \\
\hline
\end{tabular}

Table.3 Weil-Felix test results

\begin{tabular}{|l|c|}
\hline Antigen & No. of positive samples \\
\hline Only OX 19 & - \\
\hline Only OX 2 & 13 \\
\hline Only OX K & 05 \\
\hline OX 19 + OX 2 & 09 \\
\hline OX 19+ OX K & 05 \\
\hline OX 2 + OX K & 14 \\
\hline OX 19+ OX 2 + OX K & 27 \\
\hline
\end{tabular}

Although rickettsiae can be isolated from or detected in clinical specimens, serological tests, especially Weil-Felix test, still remain an indispensable tool in diagnosis. In recent years the micro immunofluorescene assay (IFA) has become the reference test. The procedure appears to be the most sensitive and specific method for the diagnosis of rickettsial infections. Hechemy et al., demonstrated $70 \%$ agreement between $\mathrm{WF}$ test and micro IF results especially with a rise in WF titre (Deepali et al., 2015). Greater clinical awareness, a higher index of suspicion, better use of available diagnostic tools would increase the frequency with which rickettsial diseases are diagnosed (Sanjay et al., 2012). A delay in diagnosis and therapy is a significant factor associated 
with death or severe illness and irreversible damage to important organs (Rajesh et al., 2009). Empirical treatment with chloramphenicol, doxycycline should be considered to reduce the high mortality and morbidity (Nigwekar et al., 2013).

In conclusion, prevalence of rickettsial diseases $73(26.35 \%)$ is significantly high, especially in children's and hence should be included in the differential diagnosis of PUO. Simple serological test (Weil-Felix test) is helpful in diagnosis. Though it is not a very sensitive test but is rather specific test and has to be interpreted in the correct clinical context.

\section{References}

Anuradha Sharma, et al. 2005. Investigation of an outbreak of Scrub typhus in the Himalayan region of India. Jpn. J. Infect. Dis., 58: 208-210.

Anuradha Sood, Smriti Chauhan. 2013. Rickettsial Diseases: An Urgent Need to Upgrade Diagnostic Facilities. Int. J. Recent Trends in Sci. Technol., 7(1): 20-21.

Deepali Danave, S.N., Kothadia. 2015. Role of Weil Felix Test for Rickettsial Infections. IOSR J. Dental and Med. Sci., 14(7): 52-54.

Ganavalli Subramanya Ajantha, et al., 2013. Rickettsiosis: A cause of acute febrile illness and value of Weil-Felix test. Indian J. Public Health, Vol 57, Issue 3: $182-183$.

Mahajan, S.K., R. Kashyap, A. Kanga, V. 2006. Sharma, B.S. Prasher, L.S. Pal. Relevance of Weil-Felix Test in Diagnosis of Scrub Typhus in India. JAPI, 54: 619-621.

Narendra rathi, Akanksha rathi. 2010. Rickettsial Infections: Indian Perspective. Indian Pediatrics, 47: 157-164.
Narendra, B.R., Akanksha, N., Rathi, Michael, H., Goodman, and Zubair, H., Aghai. 2011. Rickettsial Diseases in Central India: Proposed Clinical Scoring System for Early Detection of Spotted Fever. Indian Pediatrics, 48: 867-872.

Nigwekar, P., Kavar, Y., Shrikhande, D.Y., Ashok Kumar, C. 2013. Clinicopathological profile of rickettsial fever in a rural area of western Maharashtra, India. Pravara Med. Rev., 5(3): 5-9.

Ponugoti Munilakshmi, et al. 2015. FUO Cases Showing Prevalence of Scrub Typhus: A Comparative Study by ELISA and Rapid Test in a Tertiary Care Hospital in Andhra Pradesh, India. Int. J. Curr. Microbiol. App. Sci., 4(2): 632-640.

Prabhakaran, A. 2010. Serological Study of Rickettsial Diseases in Human and Rodent Population in Chittoor Dist. (A.P.). J. Commun. Dis., 42(3): 209213.

Raghu Kumar, K.G., Tejashree, P., Basavarajappa, K.G. 2015. Serological evidence of rickettsial infections in and around Davangere. Int. J. Med. Sci. Public Health, 4: 1731-1734.

Rajesh Joshi, Abhilasha Punde, Alpana Ohri. 2009. Rickettsial Infections seen in Rural India. Bombay Hosp. J., Vol. 51, No. 3: 385-387.

Sanjay, K., Mahajan. 2012. Rickettsial Diseases. JAPI, 60: 37-44.

Suchitra Shenoy, Shantharam Baliga, Ashok J. 2015. Rickettsial diseases in children. J. Int. Med. Dentistry, 2(1): 63-73.

Tanveer Nawab, Srinivasa, S., Sai Praneeth Reddy. 2015. A clinical study of rickettsial disease and its manifestations. Curr. Pediatr. Res., 19(1\&2): 17-20. 
Tay, S.T., M. Kamalanathan and M.Y. Rohani. 2003. Detection of rickettsial antibodies using Weil-Felix (OXK and OX19) antigens and the indirect immunoperoxidase assay. Southeast
Asian J. Trop. Med. Public Health, Vol 34 No. 1: 171-174.

Veena Mittal, et al., 2012. Serological evidence of rickettsial infections in Delhi. Indian J. Med. Res., 135: 538541.

\section{How to cite this article:}

Mita D. Wadekar and N.B. Swaroop Rani. 2016. Seroprevalence of Rickettsial Diseases in a Tertiary Care Hospital. Int.J.Curr.Microbiol.App.Sci. 5(9): 14-18. doi: http://dx.doi.org/10.20546/ijcmas.2016.509.002 